\title{
Spontaneous Cervical Epidural Hematoma Mimicking Stroke: A New Perspective on Diagnosis and Treatment
}

\author{
Alp Yurter, Paul E. Kaloostian \\ Johns Hopkins University Medical Center, Baltimore, USA \\ Email: paulkaloostian@hotmail.com
}

Received July 25, 2013; revised August 25, 2013; accepted September 3, 2013

Copyright (C) 2013 Alp Yurter, Paul E. Kaloostian. This is an open access article distributed under the Creative Commons Attribution License, which permits unrestricted use, distribution, and reproduction in any medium, provided the original work is properly cited.

\begin{abstract}
Objective: The authors report an extremely rare case of stroke-mimicking, spontaneous cervical epidural hematoma treated with tissue plasminogen activator (TPA). Case Report: We report the case of a 69-year-old female presenting with left-sided hemiparesis of the arm and leg. She was administered by TPA because she was thought to have an ischemic stroke and intracranial CT showed no hemorrhage. However, her neurological condition continued to decline, and MRI of her cervical spine revealed a large spontaneous epidural hematoma. Subsequently, the patient underwent emergency surgery. Conclusions: TPA administration to spinal epidural hematoma (SEH) patients is dangerous. Because cervical epidural hematomas can mimic stroke, the attending medical staff needs to exercise vigilance in diagnosis. In addition to the head, the spine should also be scanned prior to TPA administration.
\end{abstract}

Keywords: Tissue Plasminogen Activator (TPA); Stroke Mimic; Spontaneous Spinal Epidural Hematoma; Cervical

\section{Introduction}

For intravenous thrombolytic therapies to be effective, a correct diagnosis of acute ischemic stroke must be made within a 3 hour window from symptom onset [1]. Tissue plasminogen activator (TPA), a thrombolytic protein, is administered to patients presenting with acute cerebral ischemia [2]. Due to the difficulty in obtaining a diagnosis in the relatively short span, it is occasionally given to patients whose stroke-like symptoms manifest from other pathologies, such as epilepsy, migraine, or psychogenic illness [2]. In these patients, adverse outcomes are typically avoided because the usual underlying conditions produce transient dysfunction without acute or chronic cerebrovascular pathology [2].

However, administering TPA to a patient with CNS hemorrhage has dire consequences [1,2]. While patients with suspected intracranial hemorrhage are excluded from TPA intervention, spinal hemorrhage is not ruled out using any image modality. This is particularly problematic because a spinal epidural hematoma (SEH) can compress the spinal cord and induce symptoms mimicking ischemic stroke; though common neurological deficits associated with spinal epidural hematoma include paraparesis and quadriparesis, acute hemiparesis, which has also been documented, may cause misdiagnosis. In patients with SEH, TPA would worsen the hematoma and complicate emergency surgery [3].

The authors present an extremely rare case of spontaneous cervical epidural hematoma exacerbated by TPA administration for hemiparesis and later treated by emergency posterior cervical decompression and fusion.

\section{Case Report}

In 2012, a 69-year-old female presented to the emergency department with left-sided hemiparesis of the arm and leg, and was thought to have a stroke. Accordingly, TPA was administered after intracranial CT showed no hemorrhage (Figure 1). However, the patient's neurological condition progressively worsened after TPA administration. MRI of the cervical spine revealed a large $\mathrm{SEH}$, extending from $\mathrm{C} 1$ to $\mathrm{T} 1$, compressing the spinal cord at multiple levels (Figures 2 and 3). After obtaining emergency consent, the patient was taken to the operating room for decompression to prevent worsening of her quadriparesis. The patient underwent fluoroscopic C1-T1 posterior decompression, evacuation of the epidural hematoma, and C2-C7 posterior instrumentation with posterior lateral fusion using autograft and allograft (Figure 4). The specimen of the patient's blood clot was sent for pathology and for culture. She tolerated the procedure well and blood loss was 800 cc. Accordingly, she had received blood transfusions in the OR with appropriate 


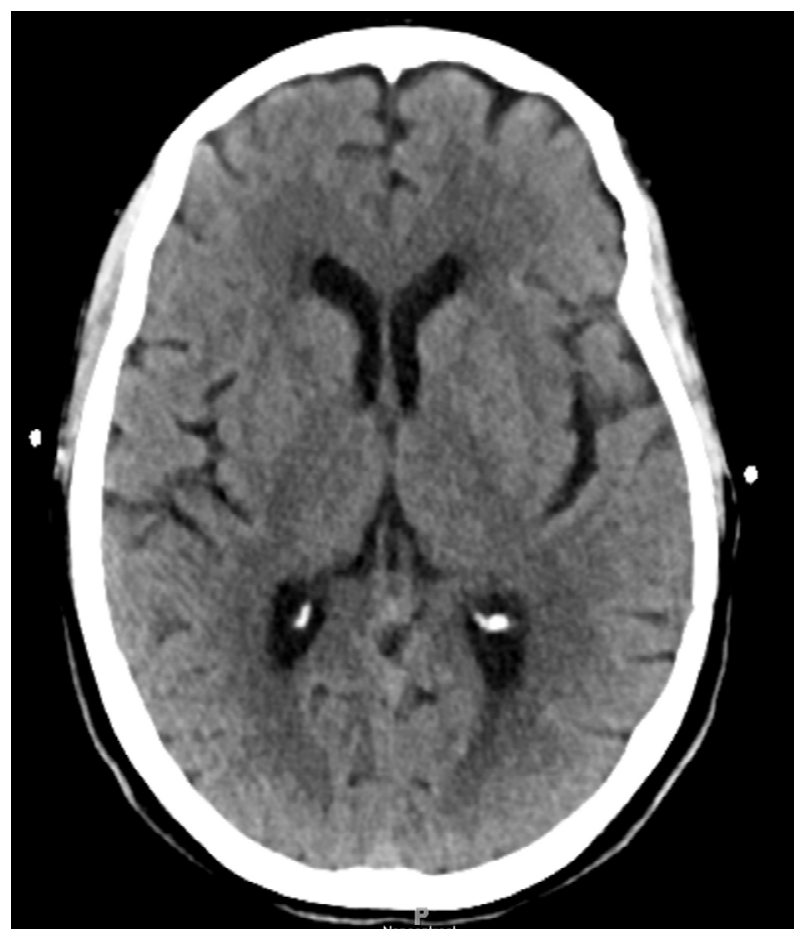

Figure 1. Preoperative intracranial CT scan without contrast reveals no hemorrhage.

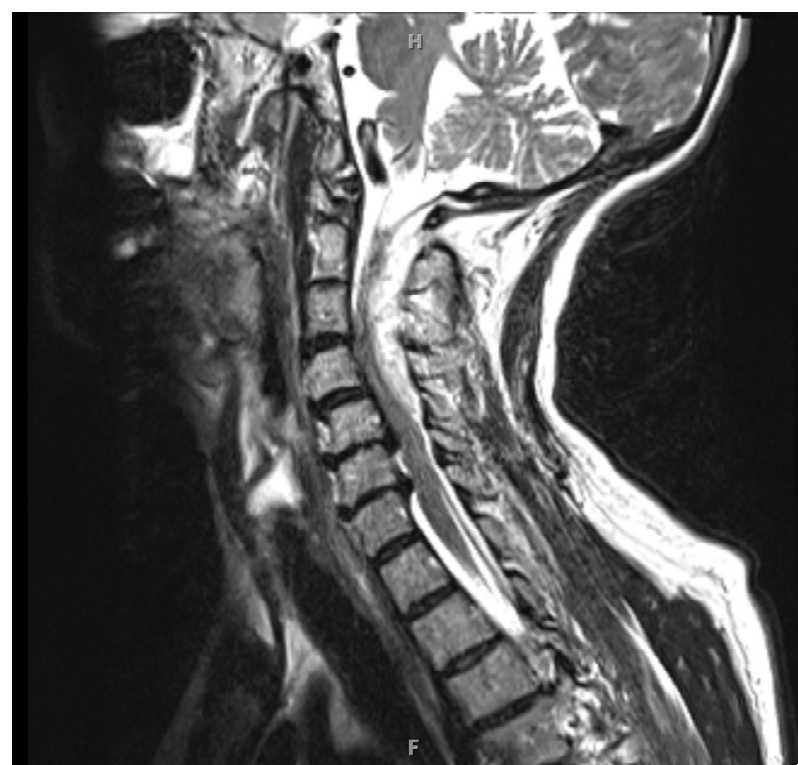

Figure 2. Preoperative sagittal T2-weighted MRI demonstrates SEH from C1-C7 generating significant mass effect estimated.

response in her hemoglobin. There were no intraoperative or postoperative complications.

Her postoperative exam was stable compared to her preoperative exam when she had left upper extremity and lower extremity weakness. She was followed by the pain resource team for pain medication management of her uncontrolled neck and upper back pain. Both of her

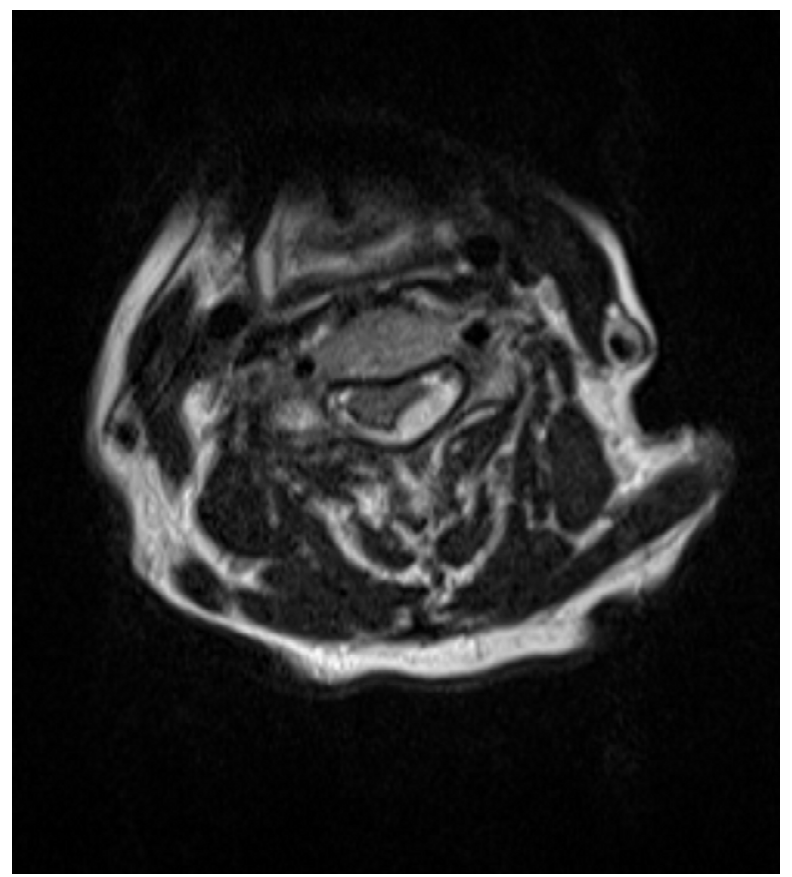

Figure 3. Preoperative axial T2-weighted MRI reveals cordcompressing SEH.

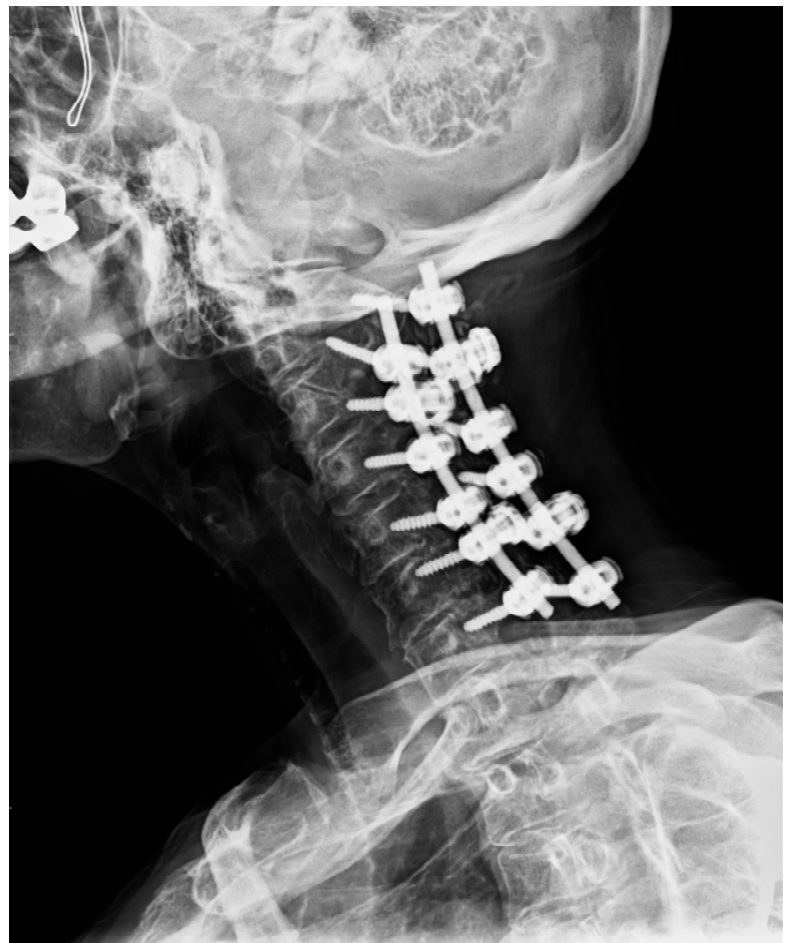

Figure 4. Postoperative oblique cervical spine $X$-ray reveals successful stabilization following laminectomy and evacuation of epidural hematoma with posterior instrumentation from C2-C7.

drains had been successfully removed without incident. Home physical therapy and home occupational therapy were arranged for the patient, as well as home skilled 
nursing, social work, and home health aide. Upon discharge, she was fully alert and oriented, and her pain was better controlled. She had 5/5 strength in her right limbs and had 4+ out of 5 strength in her left limbs. Moreover, her wound incision was clean, dry, and intact. She was stable and ready for discharge six days after being admitted.

\section{Discussion}

Most SEHs are spontaneous, and can occur from exercise, trauma, surgery, lumbar puncture, coagulopathy, vascular malformation, and chiropractic spinal manipulation [4-8]; nonspontaneous SEHs occur rarely [9]. Neurologically, patients with SEH typically present with quadriparesis and paraparesis; however, symptoms of chest pain, flank pain, monoparesis, and hemiparesis have also been documented, and are potential sources of misdiagnosis [3]. Further, cervical lesions may be indicated in the presence of sudden onset of acute neck pain, alternating hemiparesis, quadriparesis, absence of cranial nerve sign, and negative intracranial CT scans [3]. Moreover, while early surgical intervention is paramount for healthy outcomes in most, conservative treatment may be sufficient for those with early and rapid improvement in neurological function [10].

To our knowledge, there is only one other case of a patient with spontaneous SEH treated with TPA. This patient presented with progressive and fluctuating painful triparesis coupled with acute onset dissociated sensory loss. Further, the patient complained of dysarthria and transient altered mental status at the onset of symptoms, and was diagnosed with ischemic infarction of the brainstem and spinal cord accompanied by vertebral artery dissection. Because diagnosis occurred 2.5 hours after symptom onset, TPA was administered promptly. There was no change in a follow-up neurological examination so the cervical spine was scanned with MRI, revealing a hematoma from C4-T2. Emergency laminectomy and hematoma removal was performed, without major complications, and neurological improvement was noted 50 days postoperatively [1].

In our case, hemiparesis was initially attributed to ischemic stroke, a condition that requires emergent stabilization and possible thrombolytic treatment. However, thrombolytic treatment for patients with CNS hematoma is a grave error. We believe that TPA administration likely enlarged her existing hematoma, resulting in neurological decline. Therefore, prompt imaging and clinical suspicion are necessary for SEH diagnosis [11-13].

\section{Conclusion}

TPA administration to SEH patients is dangerous. Because cervical epidural hematomas can mimic stroke, the attending medical staff needs to exercise vigilance in diagnosis. In addition to the head, the spine should also be scanned prior to TPA administration.

\section{REFERENCES}

[1] S. Son, D. H. Kang, D. S. Choi, S. K. Kim, B. H. Lim and N. C. Choi, "A Case of Spontaneous Spinal Epidural Hematoma Mimicking a Stroke,” Neurologist, Vol. 18, No. 1, 2012, pp. 41-43. http://dx.doi.org/10.1097/NRL.0b013e31823d7ade

[2] J. W. Schmidley, S. Mallenbaum and K. Broyles, "Spinal Epidural Hematoma: An Important Stroke Mimic,” Acute Medicine, Vol. 12, No. 1, 2013, pp. 30-33.

[3] K. C. Liou, L. A. Chen and Y. J. Lin, "Cervical Spinal Epidural Hematoma Mimics Acute Ischemic Stroke," American Journal of Emergency Medicine, Vol. 30, No. 7, 2012, pp. 1322.e1-1322.e3.

[4] T. Abe, Y. Nagamine, S. Ishimatsu and Y. Tokuda, "Spinal Epidural Hematoma after Stretch Exercise: A Case Report," American Journal of Emergency Medicine, Vol. 27, No. 7, 2009, pp. 902.e1-902.e2.

[5] C. L. Chen, C. H. Lu and N. F. Chen, "Spontaneous Spinal Epidural Hematoma Presenting with Quadriplegia after Sit-Ups Exercise," American Journal of Emergency Medicine, Vol. 27, No. 9, 2009, pp. 1170.e3-1170.e7.

[6] J. Dimou, R. Jithoo and S. Bush, “A Patient with Delayed Traumatic Cervical Spinal Epidural Haematoma Presenting with Hemiparesis,” Journal of Clinical Neuroscience, Vol. 17, No. 3, 2010, pp. 404-405. http://dx.doi.org/10.1016/j.jocn.2009.02.030

[7] S. Lidder, K. J. Lang, S. Masterson and S. Blagg, “Acute Spinal Epidural Haematoma Causing Cord Compression after Chiropractic Neck Manipulation: An Under-Recognised Serious Hazard?” Journal of the Royal Army Medical Corps, Vol. 156, No. 4, 2010, pp. 255-257. http://dx.doi.org/10.1136/jramc-156-04-11

[8] A. M. Vitali and P. Steinbok, "Spontaneous Spinal Epidural Hematoma Following Weight Lifting," Canadian Journal of Neurological Sciences, Vol. 35, No. 2, 2008, pp. 262-264.

[9] Z. Liu, Q. Jiao, J. Xu, X. Wang, S. Li and C. You, “Spontaneous Spinal Epidural Hematoma: Analysis of 23 Cases," Surgical Neurology, Vol. 69, No. 3, 2008, pp. 253-260. http://dx.doi.org/10.1016/j.surneu.2007.02.019

[10] J. Duffill, O. C. Sparrow, J. Millar and C. S. Barker, “Can Spontaneous Spinal Epidural Haematoma Be Managed Safely without Operation? A Report of Four Cases," Journal of Neurology, Neurosurgery \& Psychiatry, Vol. 69, No. 6, 2000, pp. 816-819. http://dx.doi.org/10.1136/jnnp.69.6.816

[11] M. T. Lawton, R. W. Porter, J. E. Heiserman, R. Jacobowitz, V. K. Sonntag and C. A. Dickman, "Surgical Management of Spinal Epidural Hematoma: Relationship between Surgical Timing and Neurological Outcome," Journal of Neurosurgery, Vol. 83, No. 1, 1995, pp. 1-7. http://dx.doi.org/10.3171/jns.1995.83.1.0001

[12] J. J. Shin, S. U. Kuh and Y. E. Cho, "Surgical Manage- 
ment of Spontaneous Spinal Epidural Hematoma,” European Spine Journal, Vol. 15, No. 6, 2006, pp. 998-1004.

http://dx.doi.org/10.1007/s00586-005-0965-8

[13] R. J. Groen and H. A. van Alphen, “Operative Treatment of Spontaneous Spinal Epidural Hematomas: A Study of the Factors Determining Postoperative Outcome," Neurosurgery, Vol. 39, No. 3, 1996, pp. 494-508. 